\title{
Reversibly photochromic wood constructed by depositing microencapsulated/polydimethylsiloxane composite coating
}

\author{
Zhen Jia $^{1,2} \cdot$ Wenhui Bao ${ }^{2}$ Chengyun Tao $^{2}$. \\ Wenlong Song ${ }^{1}$
}

Received: 17 July 2021 / Accepted: 27 September 2021 / Published online: 31 October 2021

(C) The Author(s) 2021

\begin{abstract}
Photochromic wood was fabricated by coating microencapsulated photochromic material (MP)/polydimethylsiloxane composites onto wood using a simple drop-coating method. Urea-melamine-formaldehyde resin was used to microencapsulate the photochromic material (PM) via in situ polymerization. The concentration of the MP affected the photochromic property of the wood surface. The total color change $\left(\Delta E^{*}\right)$ reached 82.2 when the concentration of the composite coating is $8 \%$. Adhesion tests confirmed that the composite coating adhered firmly to the wood. This method is potentially useful for the production of functional wooden products, such as anti-counterfeiting materials and aesthetic wood.
\end{abstract}

Zhen Jia and Wenhui Bao made equal contributions to this paper.

Project funding: This work was financially supported by the National Natural Science Foundation Youth Fund of China (31800489) and the Special Fund for Research on Scientific and Technological Innovation Talents in Harbin (2017RAQXJ092). Natural Science Foundation of Heilongjiang Province of China (LH2019E070), and Postdoctoral Science Foundation funded project of Heilongjiang Province of China (LBH Z16007).

The online version is available at http://www.springerlink.com

Corresponding editor: Yu Lei.

Wenlong Song

3080839041@qq.com

1 School of Mechanical and Electrical Engineering, Northeast Forestry University, Harbin 150040, People's Republic of China

2 School of Civil Engineering, Harbin University, Harbin 150086, People's Republic of China
Keywords Wood Photochromism - Microencapsulation · Urea-melamine-formaldehyde resin $\cdot$ Drop-coating method

\section{Introduction}

Microencapsulation is used to enclose solid and liquid microparticles and gasses in inert shells, which can then be used for the sustained release of the materials within the core (Azagheswari et al. 2015). Microcapsules are classified based on their functions (Lee et al. 2016), including fire resistance (Ma et al. 2013), thermochromism (Ma and Zhu 2009), photoresponsivity (Hu et al. 2016), and energy storage (Chen et al. 2015) (Fig. 1). Microencapsulation has been used in the manufacture of several products including adhesives, agrochemicals, pharmaceuticals, and other advanced functional materials (Hu et al. 2014; Xu et al. 2016). Wood has been used by mankind for millennia owing to its excellent strength-to-weight ratio, aesthetic appearance, and low thermal expansion (Berglund and Burgert 2018). However, it is also susceptible to dimensional changes, biological attack, discoloration, and extensive checking. Hence, many modification methods have been developed to improve the functions or applications of wood products (Lang et al. 2018). Although the number of wood modification methods has greatly expanded recently, wood functionalization is still a focus of both industry and academia (Perez et al. 2018).

Photochromic materials (PMs) undergo reversible color changes when illuminated. They are used in many applications (Saputro et al. 2019), including building materials, solar cells, displays, and organic memory devices (Shallcross et al. 2013). Li et al. (2017) produced photoresponsive inorganic/organic hybrids by impregnating wood with phosphomolybdic acid/polyvinylpyrrolidone composites under pressure. Hu et al. (2015) developed photochromic plywood 
Fig. 1 Combination of functional microcapsules and wood for multi-functional materials

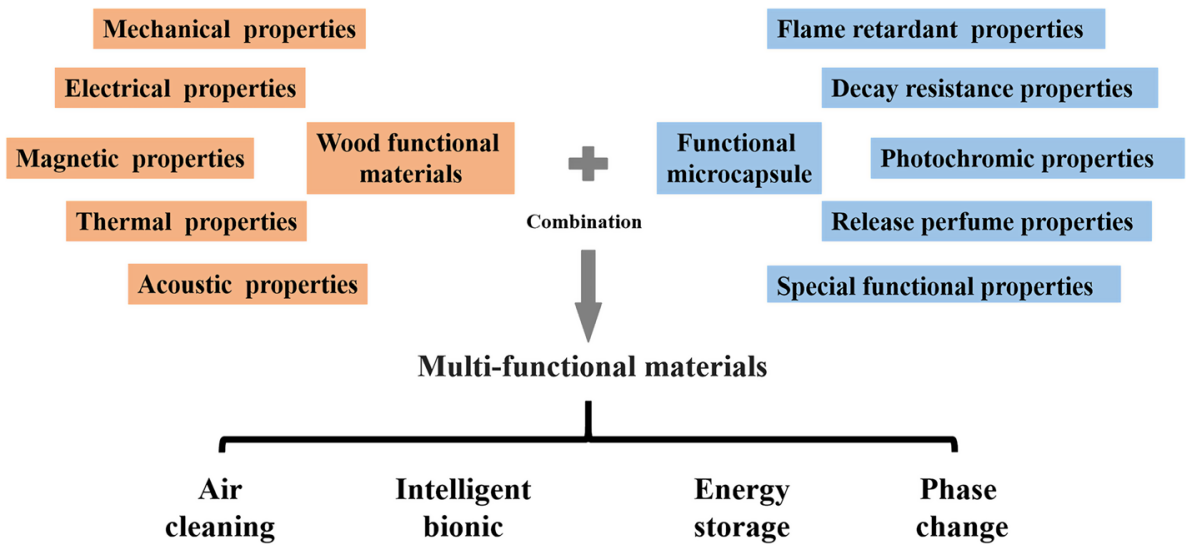

by adding photochromic microcapsules to the plywood coating. Wang et al. (2019) reported the fabrication of photochromic transparent wood by infiltrating a lignin-modified wood template with a mixture of photochromic materials to produce smart windows. Hui et al. (2015) reported the fabrication of a photoresponsive smart coating on a wood substrate, the composite had a color index of 58.2 at a PM concentration of $3 \%$ and was hydrophobic (with a contact angle of $134^{\circ}$ ). Wood-based photochromic materials have developed rapidly, but they still have some shortcomings that limit their large-scale applications (Li et al. 2010), such as complex preparation procedures, long reaction times, and poor long-term durability (Wang et al. 2014a, b).

Among the many types of PMs, the spiropyrans have attracted attention owing to their ultrafast responses, fatigue resistance and stable photochromism (Choi et al. 2003). Photochromism of spiropyran is due to photochemical conversion of the initial, the so-called closed form (colorless) or spiro (SP), form to the open form (colored) or isomeric merocyanine (MC). Upon UV irradiation such molecules are able to transform into the $\mathrm{MC}$ forms, which reverse back into the SP forms both thermally and photochemically (by irradiation with visible light). Urea-melamine-formaldehyde
(UMF) resins are generally used as microencapsulation coatings because their preparation is controllable, and they have excellent water resistance and thermal stability (Mao et al. 2013). The core-shell structure is tightly packed and tough, which prevents leakage of the core material during changes in the external environment. By encapsulation inside microcapsules, chemicals can be released at a desired rate only when and/or where the release is needed. The advantage of microencapsulated photochromic material (MP) is that the response time characteristics is significantly longer compared to the photochromic materials, mainly because of the UMF microcapsule shell act as a reflective layer to mitigation the light absorption. Polydimethylsiloxane (PDMS) is used in microfluidic devices, self-powered devices, and electronic devices owing to its low cost, optical properties, biocompatibility, and water impermeability.

Herein, we report a facile method for fabricating low-cost, mechanically-durable organic photochromic surfaces with long-term coloration on solid wood. We began by synthesizing a microencapsulated photochromic material (MP) using a UMF resin, then dropped a MP-PDMS solution onto the wood surface. The process by which the photochromic wood was fabricated is illustrated in Fig. 2. In this coating system,
Fig. 2 Schematic of the synthesis of photochromic wood
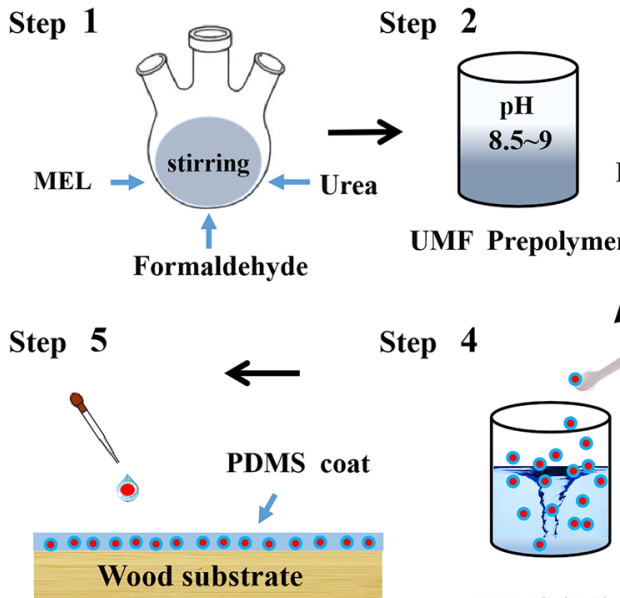

Step 4

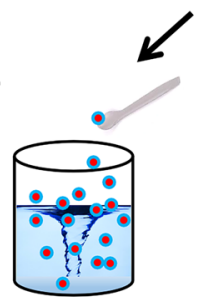

PDMS / Microcapsule solution 
PDMS was used as an elastic material that provided resistance to external damage and ensured mechanical durability (Toepke and Beebe 2006). We demonstrated the large-scale production of photochromic wood, which was subsequently used as a photo-switchable and colorful smart wood surface. We carried out a series of characterizations and measurements to determine the properties of the films. The surface morphologies and chemical compositions were characterized by scanning electron microscopy (SEM), atomic force microscopy (AFM), X-ray photoelectron spectroscopy (XPS), and attenuated total reflectance Fourier-transform infrared spectroscopy (ATR-FTIR).

\section{Materials and methods}

\section{Materials}

Wood samples obtained from a forest in Harbin, P. R. China were cut into specimens measuring $50 \mathrm{~mm} \times 25 \mathrm{~mm} \times 5 \mathrm{~mm}$ (length $\times$ width $\times$ height). After ultrasonically rinsing in ethanol and deionized water for $10 \mathrm{~min}$, the wood specimens were oven-dried $\left(24 \mathrm{~h}, 103{ }^{\circ} \mathrm{C} \pm 2{ }^{\circ} \mathrm{C}\right)$ to a constant weight, which was recorded. The photochromic material 3-(2,4-dimethoxyphenyl)-3-(4-methoxyphenyl)-3Hnaphthol[2,1-b]pyran (95.0\%) was obtained from Sigma Chemical Reagent Corporation, Darmstadt, Germany. Urea, melamine, and formaldehyde were purchased from Tianjin Chemical Reagents Co., Ltd., China. PDMS (Sylgard 184) and the curing agent were provided by Dow Corning Co., Ltd., USA.

\section{Sample preparation}

\section{Preparation of the microencapsulated photochromic material.}

Synthesis of prepolymer: Urea, melamine, and 37\% formaldehyde solution were placed in a three-neck flask with a stirrer. First, the $\mathrm{pH}$ of the mixture was adjusted to 8.5-9.0 with $20 \% \mathrm{NaOH}$ solution. The mixture was then heated to $75^{\circ} \mathrm{C}$ and held at that temperature for $10 \mathrm{~min}$ (Kandelbauer et al. 2007). Second, $20 \mathrm{~g}$ of urea was added to the mixture, which was heated to $80{ }^{\circ} \mathrm{C}$ for $20 \mathrm{~min}$ and held at $\mathrm{pH} 7.5$ at a constant temperature (No and Kim 2007). Finally, $40 \mathrm{~g}$ of urea was added to the mixture, which was cooled to $60{ }^{\circ} \mathrm{C}$ and held at a constant temperature for $30 \mathrm{~min}$ to obtain a prepolymer (Wang et al. 2014a, b).

A mixture of the photochromic material $(3 \mathrm{~g})$ and ethanol $(20 \mathrm{~mL})$ was stirred at $1000 \mathrm{rpm}$ at $30^{\circ} \mathrm{C}$ for $10 \mathrm{~min}$. Then, after adding the UMF prepolymer, the $\mathrm{pH}$ of the mixture was adjusted to 4-5 with 20\% acetic acid (No and Kim 2004). The resulting mixture was heated at $80{ }^{\circ} \mathrm{C}$ for $3 \mathrm{~h}$ while stirring. After cooling to room temperature, the product was filtered, washed with distilled water, and dried at $105^{\circ} \mathrm{C}$ to obtain the MP powder.

\section{Preparation of the photochromic wood}

First, a base coat solution of PDMS was prepared by dissolving $5 \mathrm{~g}$ of PDMS and $0.5 \mathrm{~g}$ of curing agent in $35 \mathrm{~g}$ of butyl acetate solution while stirring magnetically. Next, MP was added to the PDMS solution, which was magnetically stirred at $45^{\circ} \mathrm{C}$ for $2 \mathrm{~h}$, and ultrasonically dispersed at room temperature for $30 \mathrm{~min}$. The percentage of MP was pre-set at $0,2,4 \%, 6$ and $8 \%$. Finally, the wood was dipped in the MP-PDMS solution for $10 \mathrm{~min}$ and cured at $80{ }^{\circ} \mathrm{C}$ for $5 \mathrm{~h}$ to obtain the PDMS pre-coated substrate.

\section{Characterization}

The morphologies of the as-prepared samples were investigated by SEM (Quanta 200, FEI, Eindhoven, Holland; $12.5 \mathrm{kV}$ ). The chemical compositions of treated and untreated wood were determined by FTIR (Magna-IR 560, Nicolet) and X-ray photoelectron spectroscopy (K-Alpha; ThermoFisher Scientific Company, Massachusetts, USA). Non-contact surface profilometry was used to determine the surface morphology. Thermogravimetric (TG) analysis was performed on a TA-50 instrument under an $\mathrm{N}_{2}$ atmosphere. The solar simulator (XES-40S2-CE, Lamp L150SS, Japan, the wave length of solar light: $300-1100 \mathrm{~nm}$ ) was used to examine the stable UV light and VIS light, and the irradiance was the standard level of $1000 \mathrm{Wm}^{-2}$. The UV light and VIS light were transformed each other by controlling the UV filter (300-380 nm) and VIS filter (380-780 nm), respectively. The distance between the lamp and samples was $20 \mathrm{~cm}$. The sample color was determined using an optical spectrophotometer (CM-2300d; Konica Minolta Sensing, Inc.), and the color change was evaluated using $L, a$, and $b$ color parameter values (Gan et al. 2015). The total color changes $(\Delta E)$ of the sample coatings were calculated using Eq. 1:

$\Delta E=\sqrt{\Delta a^{2}+\Delta b^{2}+\Delta L^{2}}$

\section{Results and discussion}

\section{Physical and chemical properties of the microcapsules}

The structure of the microcapsules was determined by SEM. As shown in Fig. 3a, the PM comprised microspheres with diameters ranging from 1 to $5 \mu \mathrm{m}$ with relatively smooth globular surfaces. The PM was completely covered with the cured UMF resin shell to form the MP. As expected, most 

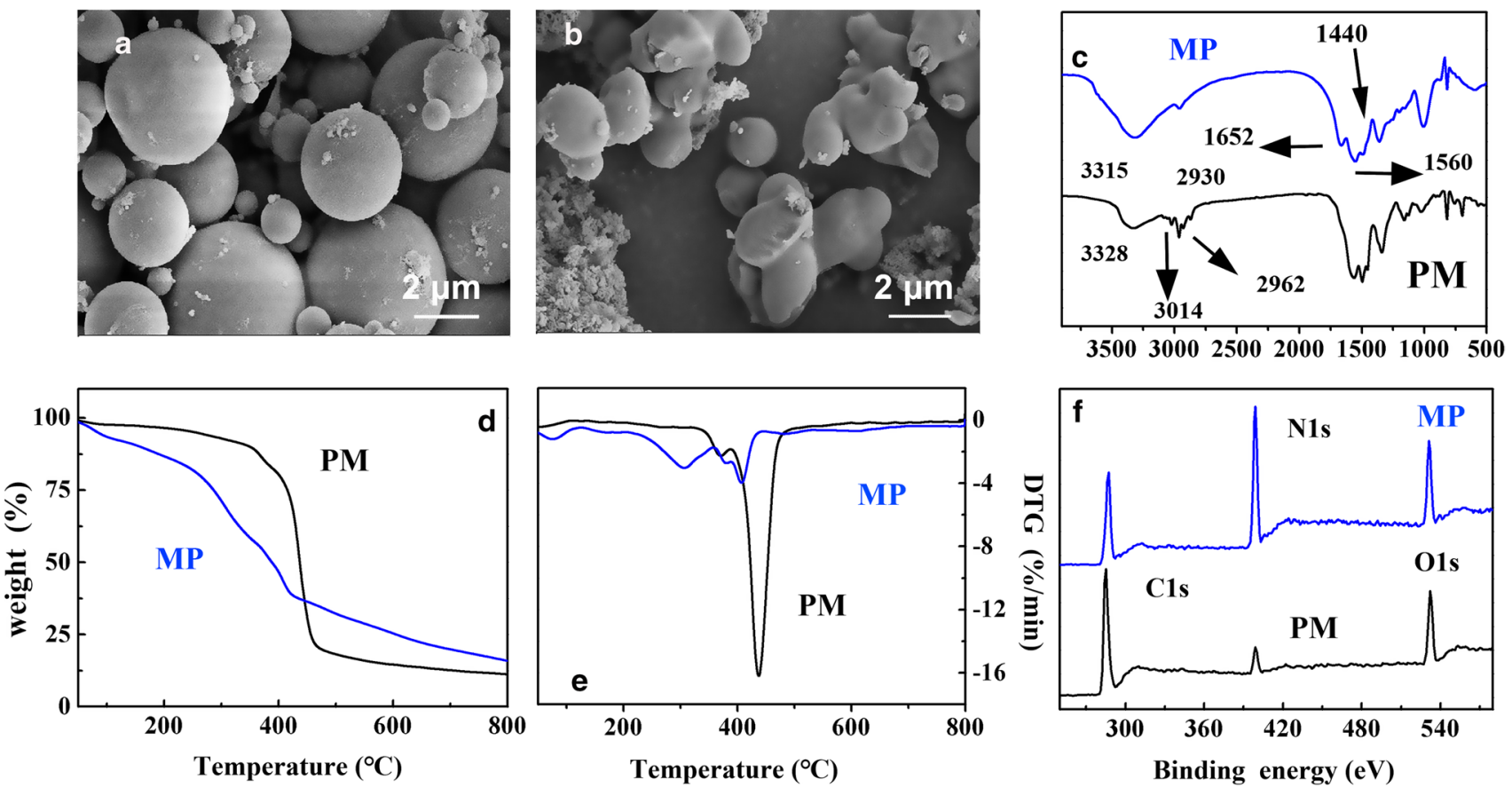

Fig. 3 Scanning electron microscopy (SEM) investigation of surface morphology: (a) photochromic material (PM), and (b) microencapsulated photochromic material (MP); (c) Fourier-transform infrared

spectroscopy (FTIR) spectra of PM and MP; (d) TG and (e) DTG curves of PM and MP; (f) X-ray photoelectron spectroscopy (XPS) spectra of PM and MP

of the visible MP was polypore, with highly heterogeneous particle sizes and shapes (Fig. 3b).

The FTIR spectra of the PM and MP are presented in Fig. 3c. The typical absorption peak of the PM at $3023 \mathrm{~cm}^{-1}$ was attributed to the aromatic $\mathrm{C}-\mathrm{H}$ stretching vibration (Kim et al. 2008). The absorption peaks at 2962 and $2930 \mathrm{~cm}^{-1}$ were attributable to alkane C-H stretching vibrations. The strong and wide absorption peak at $3346 \mathrm{~cm}^{-1}$ was attributable to the ring vibration of melamine in the UMF resin (Pakdel et al. 2007). The band at $1652 \mathrm{~cm}^{-1}$ was associated with the $\mathrm{C}=\mathrm{O}$ stretching vibrations of the amide group of methyl urea and the $\mathrm{C}=\mathrm{N}$ stretching vibrations of the triazine ring (Chai et al. 2018). The peak at $1560 \mathrm{~cm}^{-1}$ was attributable to a combination of the vibrations of $\mathrm{C}-\mathrm{N}$ and $-\mathrm{NH}_{2}$ in the amide and melamine (Benson 2003). The band at $1440 \mathrm{~cm}^{-1}$ was indicative of amide and triazine stretching vibrations (Marvel et al. 1946). The surface elemental compositions of carbon, oxygen, and nitrogen in the PM and MP were determined by XPS (Fig. 3f). The peaks located at 294, 408, and $541 \mathrm{eV}$ were attributed to $\mathrm{C} 1 \mathrm{~s}, \mathrm{O} 1 \mathrm{~s}$, and $\mathrm{N} 1 \mathrm{~s}$, respectively. The MP showed a significant increase in the nitrogen concentration compared with the PM, whereas the oxygen content remained nearly constant. The MP N/C ratio determined by elemental analysis was 0.863 , which was higher than the $\mathrm{PM}$ N/C ratio of 0.123 . This indicated that that the PM was well-coated by the resin (Coullerez et al. 2000).

The TG and differential thermogravimetric (DTG) curves of the PM and MP shown in Fig. 3d, e reveal that the PM began to decompose at approximately $310^{\circ} \mathrm{C}$ and decomposed almost completely at approximately $440{ }^{\circ} \mathrm{C}$. The residual weight of the $\mathrm{PM}$ was $11.6 \%$ at $800{ }^{\circ} \mathrm{C}$. Compared with PM, MP has two main decomposition processes. The initial decomposition stage of the MP occurred more quickly than the decomposition of the PM owing to water evaporation and slow free formaldehyde emission (Wu et al. 2008). During the second decomposition stage (above $425{ }^{\circ} \mathrm{C}$ ), the MP was more stable than the PM because the UMF resin produced incombustible gases, such as $\mathrm{NH}_{3}$ and $\mathrm{CO}_{2}$, which formed a thermally stable honeycomb char (Siimer et al. 2009). The $T_{\max }$ values for the two MP decomposition stages were 305 and $410{ }^{\circ} \mathrm{C}$ (Németh et al. 2018). After decomposition at $800{ }^{\circ} \mathrm{C}$, the MP left approximately $23.5 \%$ residue, which was much more than the PM (Li et al. 2007). Therefore, the MP was more effective at improving the thermal stability than the PM. As mentioned above, the results further indicated that the PM was completely covered with the cured UMF resin shell to form the MP.

\section{Morphology of the photochromic wood surface}

The surface morphologies of the pristine wood and the MP-PDMS nanocomposite-coated wood are presented in Fig. $4 \mathrm{a}$, d. The original wood surface comprised a 


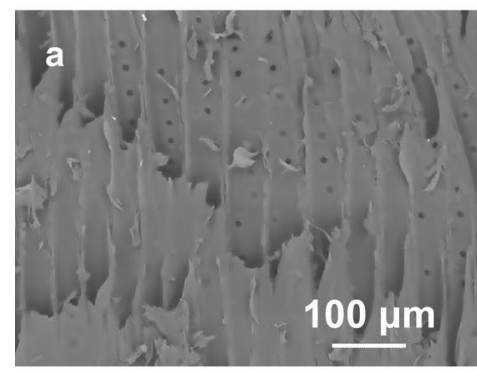

\section{b}
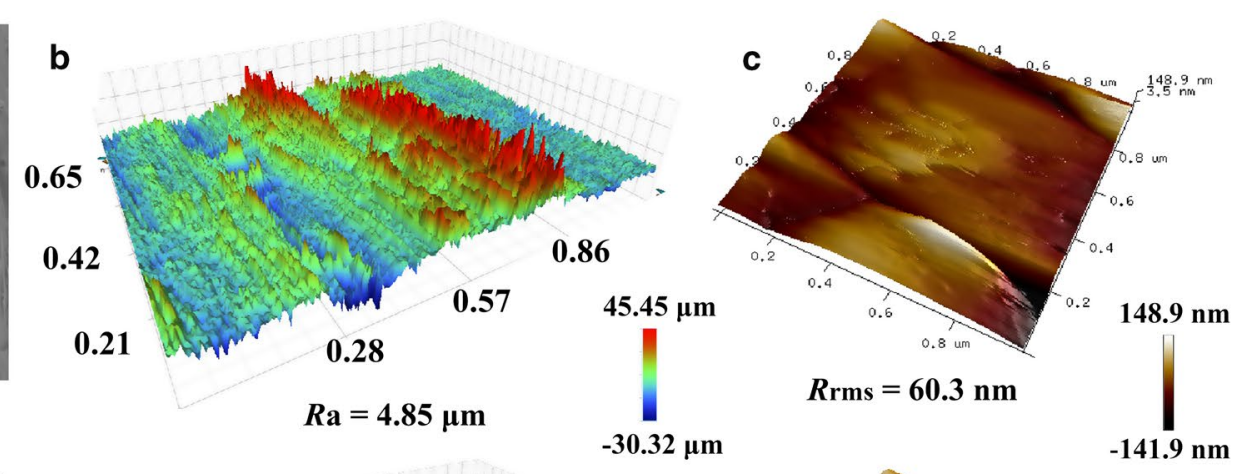

$R \mathrm{rms}=60.3 \mathrm{~nm}$

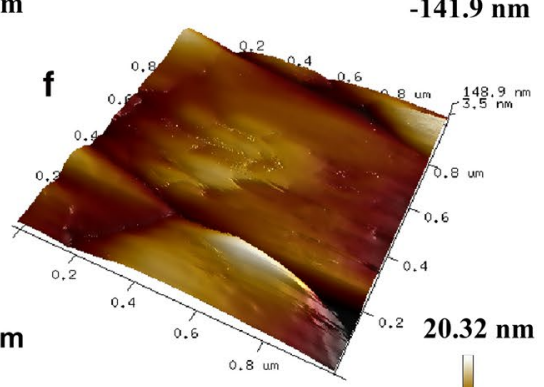

$R \mathrm{rms}=27.9 \mathrm{~nm}$

e

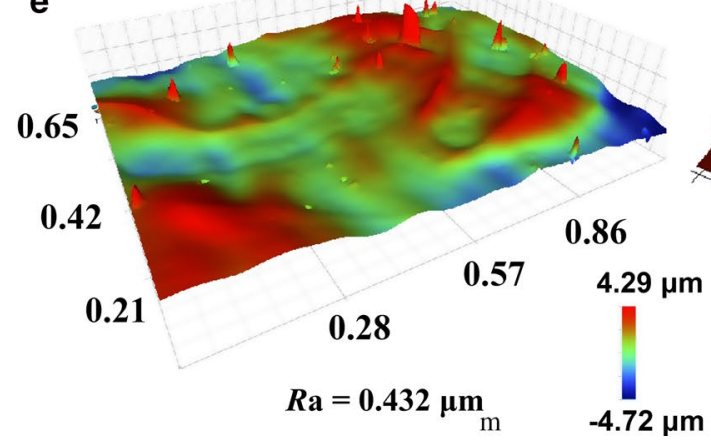

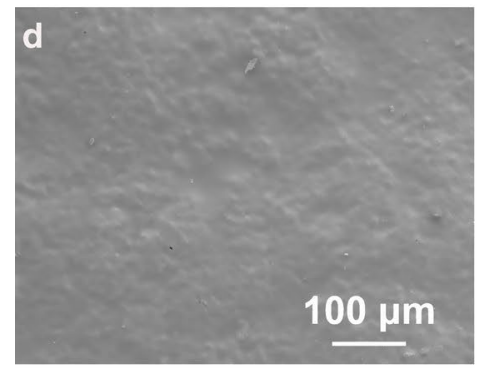

Fig. 4 Scanning electron microscopy (SEM) images, 3D scans, and AFM images of the pristine wood (a, b, c) and MP-PDMS nanocompositecoated $\operatorname{wood}(\mathbf{d}, \mathbf{e}, \mathbf{f})$

heterogeneous porous material with a micro-grooved surface (Fig. 4a). After dipping in the MP-PDMS coating solution, a continuous polymer film was formed on the surface of the wood (Fig. 4d). Micro-scale protrusions comprising the MP were distributed throughout the film. The MP played an important role in the photochromism, and the texture of the wood remained visible because the film was transparent.

The samples were also investigated by non-contact surface profilometry and AFM to determine changes in the surface morphology. Figure $4 \mathrm{~b}$, e presents $3 \mathrm{D}$ images of the as-prepared samples. As shown in Fig. 4b, the original wood surface was almost completely covered with a clear vertical gradient due to the complex surface texture. Compared with the original wood surface, the MP-PDMS nanocomposite-coated wood surface (Fig. 4e) was smooth and flat. The surface roughness $(R \mathrm{a})$ values were $4.85 \mu \mathrm{m}$ (original wood) and $0.432 \mu \mathrm{m}$ (MP-PDMS wood).

Tapping mode AFM images of the surface topography of the original wood and the MP-PDMS wood are shown in Fig. 4c, f. The different surface roughness values are reflected in the $R_{\mathrm{rms}}$ values ( $\mathrm{rms}=$ root mean square $=$ the standard deviation of the $Z$ value; $Z$ is the total height range analyzed) of the two surfaces, which were $60.3 \mathrm{~nm}$ (original wood) and $27.9 \mathrm{~nm}$ (MP-PDMS wood).

\section{Photochromic properties of samples with various MP concentrations}

Figure 5 shows the color parameters of the pristine wood and the wood coated with MP-PDMS with MP concentrations of 2, 4, 6 and $8 \%$ in weight. As the MP concentration increased, the lightness factor $L^{*}$ of the samples under UV irradiation decreased from 60.1 to 12.2 , indicating that the surface color darkened. The $a^{*}$ value increased markedly from 5.2 to 10.3 , implying the surface color turned a deeper shade of red. The $b^{*}$ value decreased from 13.0 to -22.3 , indicating that the surface color changed from yellow to blue. Furthermore, the total color change $\left(\Delta E^{*}\right)$ increased from 3.7 to 80.2 as the MP concentration increased from 0 to $8 \%$, confirming the photoresponsivity of the MP-PDMS-coated wood.

As shown in Fig. 6a, the lightness and chroma of the wood coated with MP-PDMS with MP concentrations of $2 \%$ in weight was very similar compared to the pristine wood. With an increase in the MP concentrations from 4 to $8 \%$ in weight, the lightness of the treated wood surface is gradually increased due to the microcapsule shell (UMF) is white (Fig. 6b-d). However, the natural color texture of the wood surface is not affected by the composite coating. After UV light irradiation, surface color slightly darker (Fig. 6e). With increasing MP concentrations, the surface color turned dark 

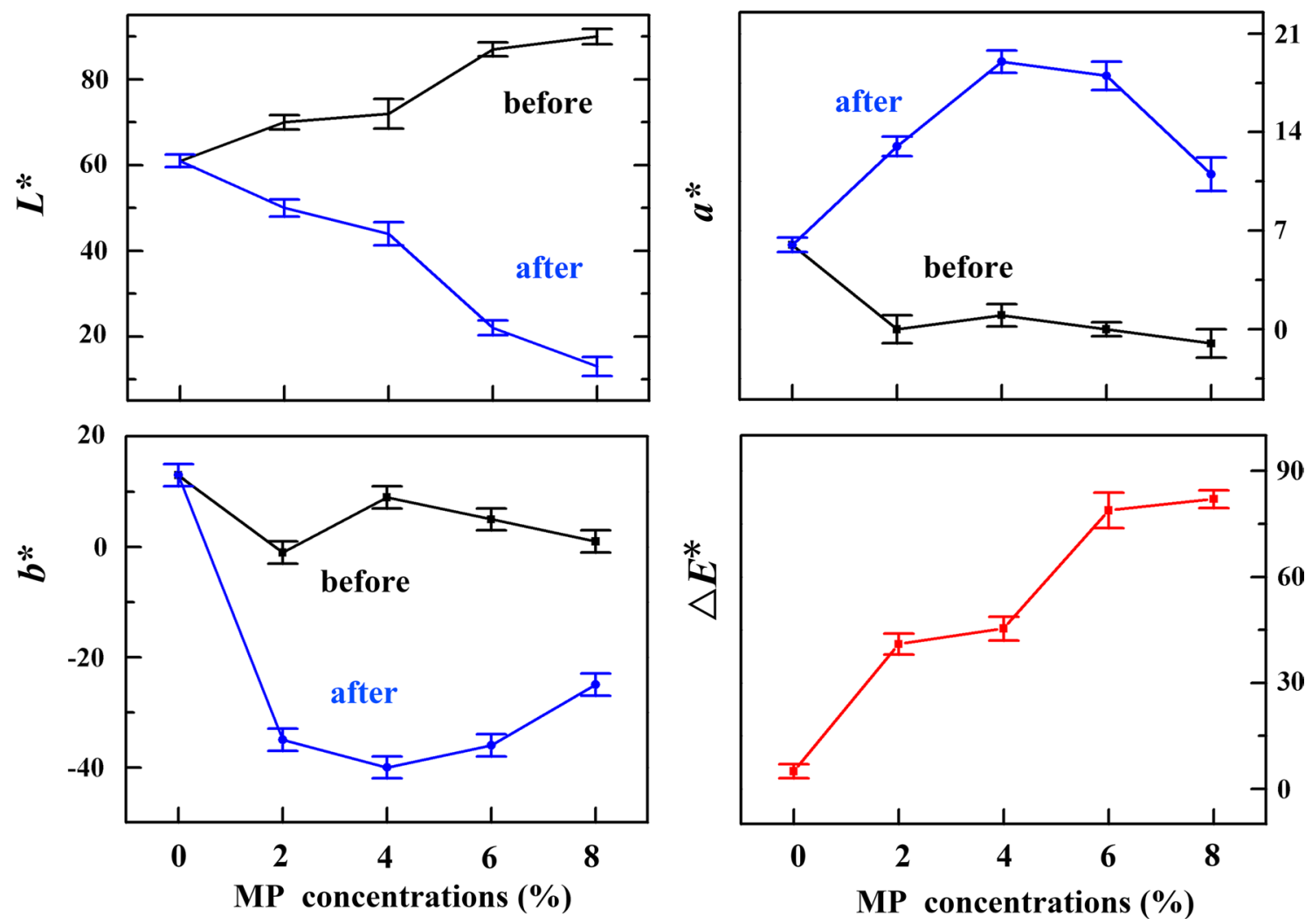

Fig. 5 Changes in the sample surface chromaticity indexes $L^{*}, b^{*}, a^{*}$, and $\Delta E^{*}$ before and after UV irradiation

red (Fig. 6f-h). Color changes of the photochromic coatings were related to the increasing number of chromophores. To further explore the relationship between the color change of the photochromic coating and the intensity of the UV irradiation, MP-wood samples with $8 \%$ concentration in weight under different UV intensity irradiation the digital images (Fig. 6a-d) were utilized. As a rule, high intensity UV irradiation leads to further increase of color intensity due to the accumulation of the open MC forms.

The practical applications of photochromic wood samples are directly affected by the response time, which includes the chromogenic time and the fading time. Figure 7 shows the response time curves of $2 \%-8 \%$ MP-wood. At a constant temperature, the response time of the sample gradually increased as the MP concentration increased, which is attributed to the fact that an increase in the MP concentration results in strong electrostatic interaction between the MP molecules and an increase in the degree of aggregation, as shown in Fig. 7a. As the concentration of the spiropyran increases, a transformation of the chemical structure is less likely because the closed form of the spiropyran increases steric hindrances; hence, there is a lag in the response time of MP-wood formed with higher MP concentrations. As a result, at high concentrations, the photochromic microcapsules samples are more stable under changing temperature conditions. As the temperature increased, the chromogenic time decreased. When the temperature exceeded $30{ }^{\circ} \mathrm{C}$, the MP concentration exerted a minimal influence on the fading time (Fig. 7b). The result for the response time is attributed to the fact that an increase in the temperature favors the breakup and recombination of the $\mathrm{C}-\mathrm{O}-\mathrm{C}$ bond in the spiropyrane, thereby increasing the conversion rate of spiropyrane to merocyanine.

\section{Color stability and adhesion properties of samples}

Figure 8a illustrates the $\Delta E$ cycle characterization of samples with various MP concentrations. The results indicate that the photoresponsivity of the MP-wood was not affected by $100 \mathrm{UV}$ irradiation cycles. To assess the mechanical resistance of the MP-wood, samples were abraded with sand and sandpaper. The surfaces of the MP-wood samples were subjected to impingement with $15 \mathrm{~g}$ of sand from a height of $25 \mathrm{~cm}$, the sand grains had diameters in the range 200-250 $\mu \mathrm{m}$. Figure 8b shows that an as-prepared sample with a $100 \mathrm{~g}$ weight on its top was placed face-down on the sandpaper and moved $50 \mathrm{~cm}$. After 100 sand impact tests, the surfaces of the films appeared smooth, with no signs 
Fig. 6 Digital images of MPwood with different concentration of 2, 4, 6, 8\% before $(\mathbf{a}-\mathbf{d})$ and after UV irradiation $(\mathbf{e}-\mathbf{h})$, MP-wood with $8 \%$ concentration after different UV intensity irradiation for (A) $50 \mathrm{Wm}^{-2}$, (B) $200 \mathrm{Wm}^{-2}$, (C) $500 \mathrm{Wm}^{-2}$, and (D) $800 \mathrm{Wm}^{-2}$
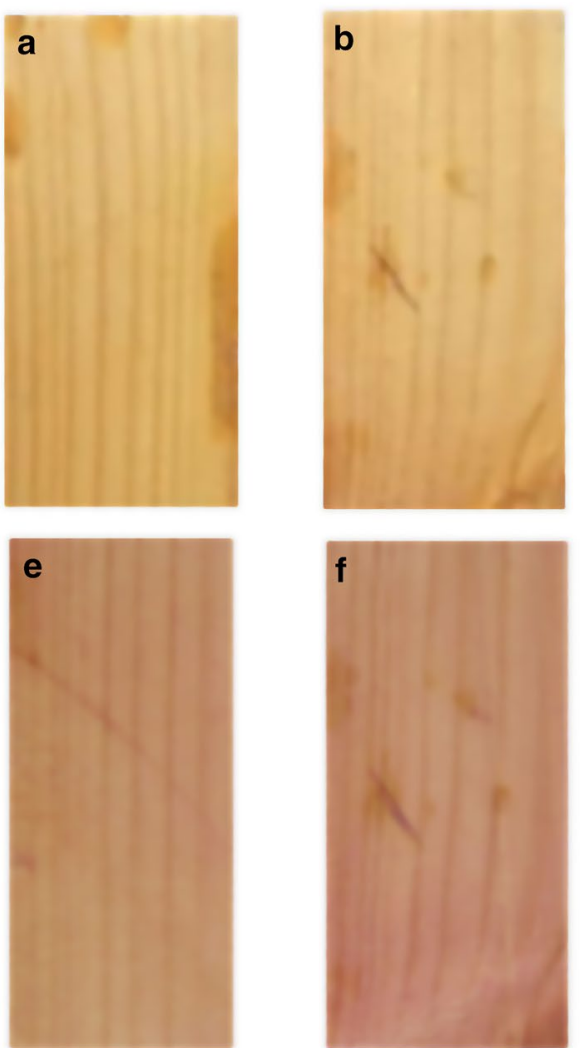

MP-2\%

\section{A}
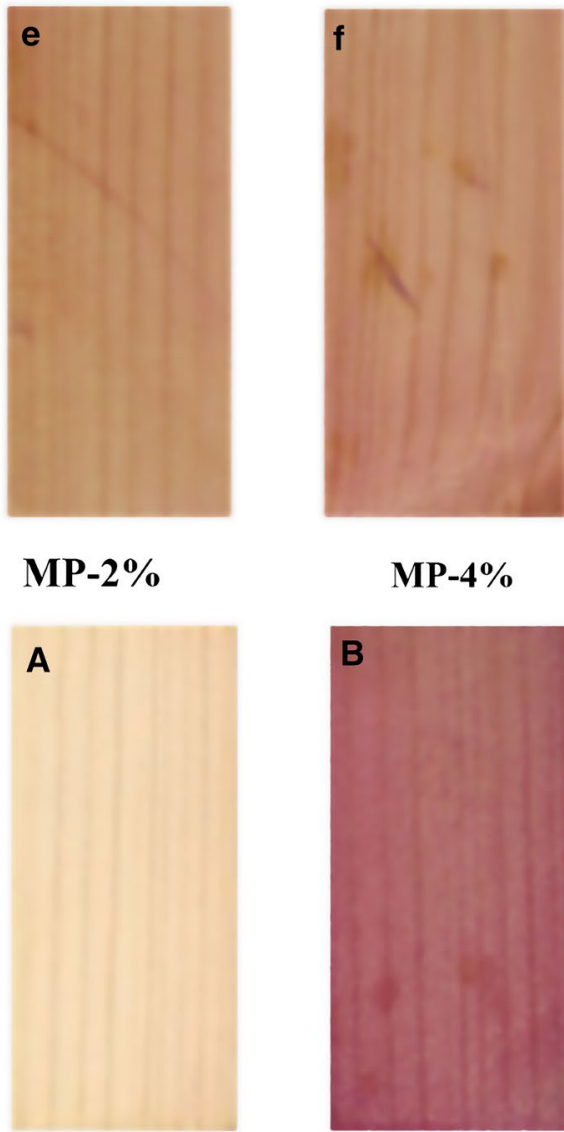

MP-4\%

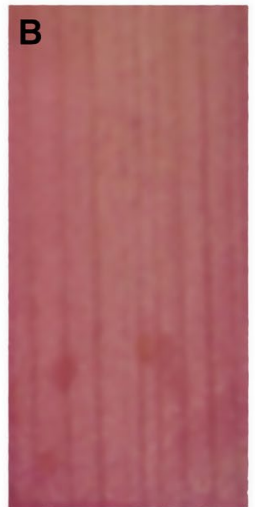

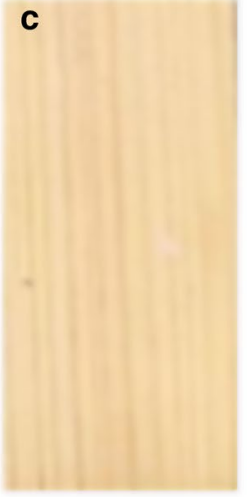
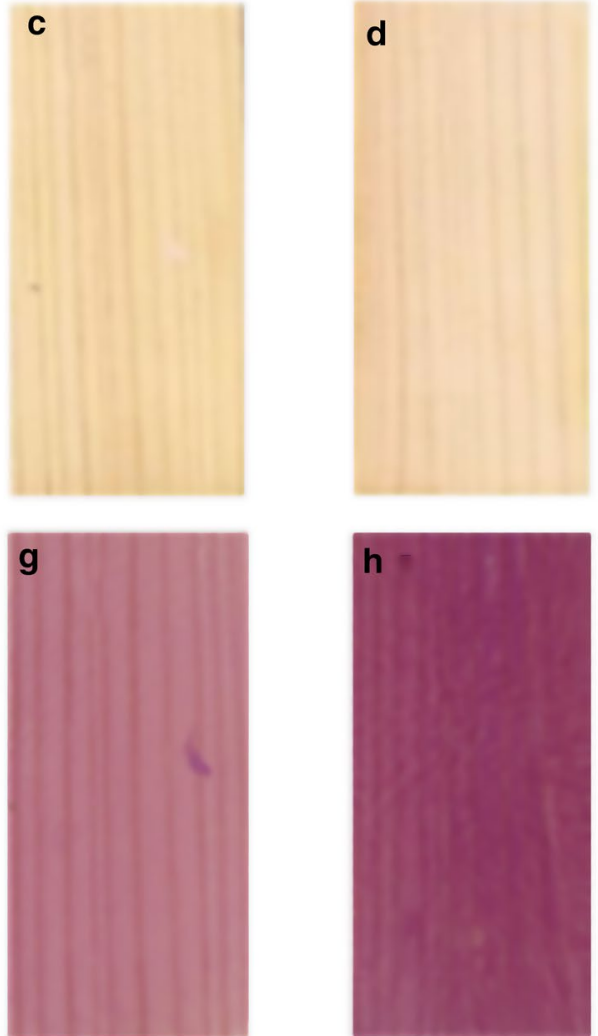

MP-6\%

MP-8\%
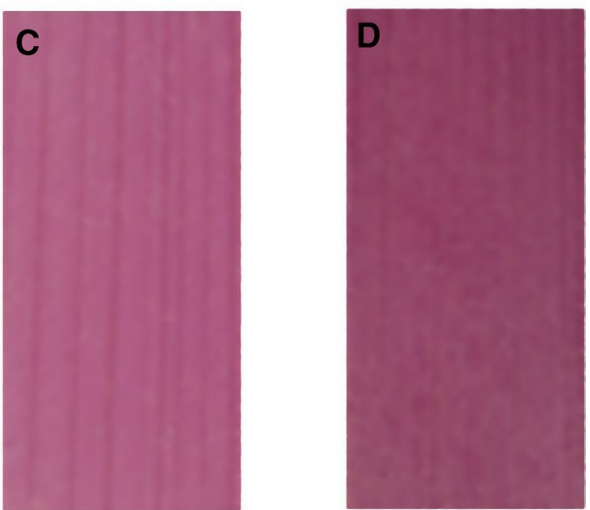

of damage (Fig. 8e). In contrast, the SEM images showed that the film was broken and severely worn because the external load of the sandpaper exceeded the strength of the film (Fig. 8f). Figure $8 \mathrm{~d}$ shows the $\Delta E^{*}$ values of the samples made with various MP concentrations (2, 4, 6 and 8\%) before and after UV aging. The sample with an MP concentration of $8 \%$ had the highest $\Delta E^{*}$ value during the aging test, indicating that UV aging resistance was proportional to the MP concentration. $\Delta E^{*}$ decreased significantly from 100 to $150 \mathrm{~h}$. The photochromic performances of the samples degraded with UV exposure time, but the samples with high MP concentrations still exhibited satisfactory photochromic performances after 250-h UV aging.

\section{Conclusion}

Photochromic films were deposited on the surface of wood by drop-coating a solution containing MP particles and PDMS. The $\Delta E^{*}$ values of the as-generated wood 


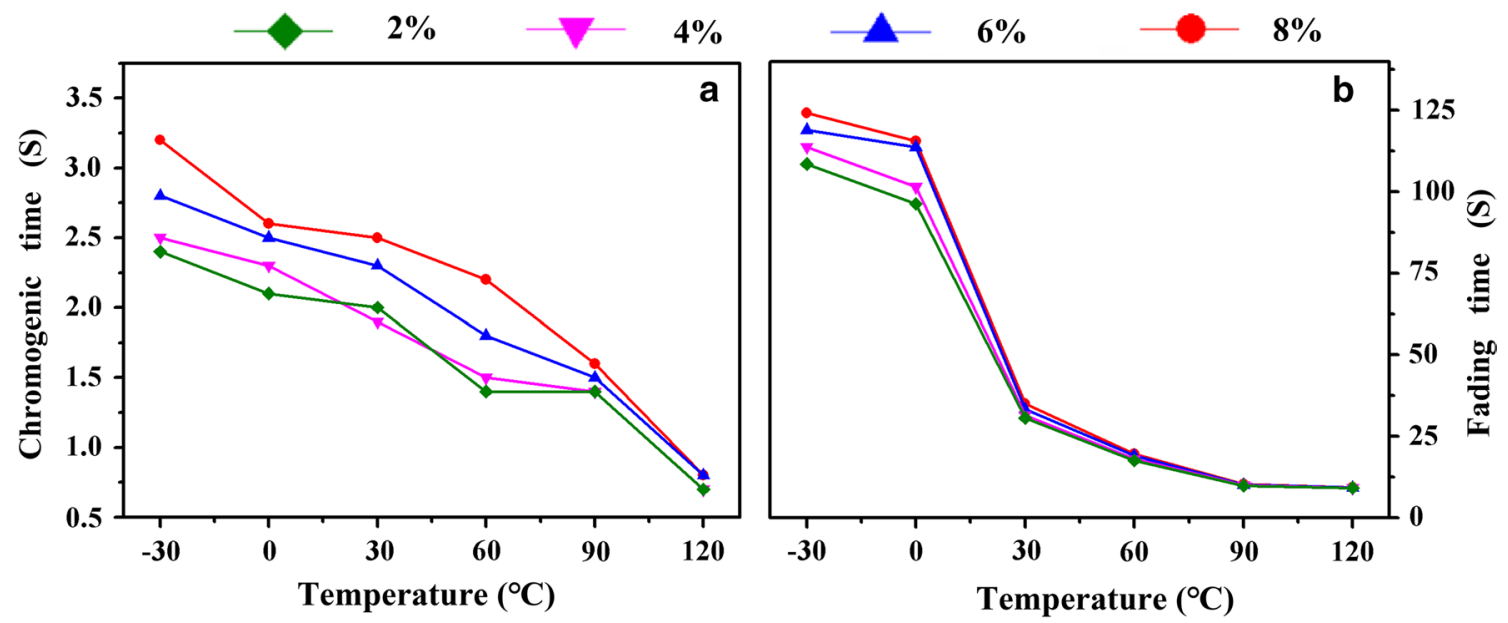

Fig. 7 Response time-temperature curves of the samples: (a) chromogenic time, and (b) fading time
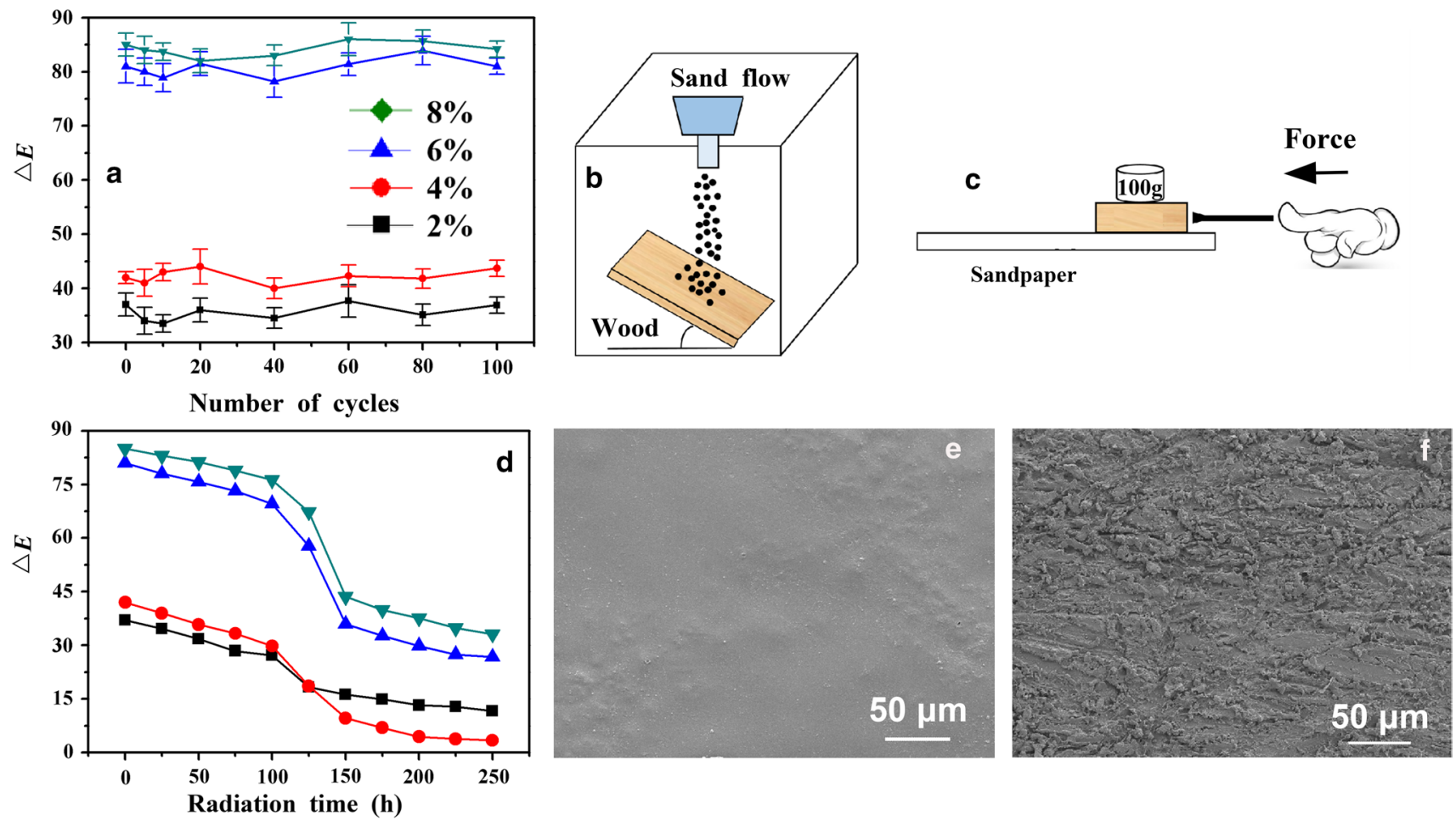

Fig. 8 (a) Cyclic UV aging tests and (d) UV aging tests on the samples. Schematic drawing of the sand abrasion test (b) and the sandpaper abrasion test (c), SEM images of the surfaces after (e) 100 cyclic impingements, (f) $50 \mathrm{~cm}$ abrasion on sandpaper

increased with increasing MP concentration, and $8.0 \%$ MP produced a photochromic surface with a $\Delta E^{*}$ of 82.2 . The chromogenic time and fading time of the photochromic coatings decreased with the increasing of temperature. The sand abrasion test revealed that the film had high mechanical resistance; however, the sandpaper abrasion test destroyed the film when the external force was higher than its own load. The present study provides an effective and convenient method for obtaining novel photochromic wood products. 
Open Access This article is licensed under a Creative Commons Attribution 4.0 International License, which permits use, sharing, adaptation, distribution and reproduction in any medium or format, as long as you give appropriate credit to the original author(s) and the source, provide a link to the Creative Commons licence, and indicate if changes were made. The images or other third party material in this article are included in the article's Creative Commons licence, unless indicated otherwise in a credit line to the material. If material is not included in the article's Creative Commons licence and your intended use is not permitted by statutory regulation or exceeds the permitted use, you will need to obtain permission directly from the copyright holder. To view a copy of this licence, visit http://creativecommons.org/licenses/by/4.0/.

\section{References}

Azagheswari BK, Padma S, Priya SP (2015) A review on microcapsules. Glob J Pharmacol 9(1):28-39

Benson MT (2003) Density functional investigation of melamine-formaldehyde cross-linking agents. 1. Partially substituted melamine. Ind Eng Chem Res 42(18):4147-4155

Berglund LA, Burgert I (2018) Bioinspired wood nanotechnology for functional materials. Adv Mater 30(19):e1704285

Chai YQ, Zhao TB, Gao X, Zhang JJ (2018) Low cracking ratio of paraffin microcapsules shelled by hydroxyl terminated polydimethylsiloxane modified melamine-formaldehyde resin. Coll Surf A 538:86-93

Chen ZH, Wang J, Yu F, Zhang ZG, Gao X (2015) Preparation and properties of graphene oxide-modified poly(melamine-formaldehyde) microcapsules containing phase change materials n-dodecanol for thermal energy storage. J Mater Chem A 3(21):11624-11630

Choi DH, Ban SY, Kim JH (2003) Stability of photochromism in new bifunctional copolymers containing spiropyran and chalcone moiety in the side chain. Bull Korean Chem Soc 24(4):441-445

Coullerez G, Léonard D, Lundmark S, Mathieu HJ (2000) Xps and tof-sims study of freeze-dried and thermally cured melamineformaldehyde resins of different molar ratios. Surf Interf Anal 29(7):431-443

Gan W, Gao L, Sun Q, Jin C, Lu Y, Li J (2015) Multifunctional wood materials with magnetic, superhydrophobic and anti-ultraviolet properties. Appl Surf Sci 332:565-572

Hu L, Lyu SY, Fu F, Huang JD (2016) Development of photochromic wood material by microcapsules. BioResources 11(4):9547-9559

Hu L, Lyu SY, Fu F, Huang JD, Wang SQ (2015) Preparation and properties of multifunctional thermochromic energy-storage wood materials. J Mater Sci 51(5):2716-2726

Hu X, Huang Z, Zhang Y (2014) Preparation of CMC-modified melamine resin spherical nano-phase change energy storage materials. Carbohydr Polym 101:83-88

Hui B, Li Y, Huang Q, Li G, Li J, Cai L, Yu H (2015) Fabrication of smart coatings based on wood substrates with photoresponsive behavior and hydrophobic performance. Mater Des 84:277-284

Kandelbauer A, Despres A, Pizzi A, Taudes I (2007) Testing by fourier transform infrared species variation during melamine-urea-formaldehyde resin preparation. J Appl Polym Sci 106(4):2192-2197

Kim CW, Oh SW, Kim YH, Cha HG, Kang YS (2008) Characterization of the spironaphthooxazine doped photochromic glass: the effect of matrix polarity and pore size. J Phys Chem C 112(4):1140-1145

Lang AW, Li Y, De Keersmaecker M, Shen DE, Osterholm AM, Berglund L, Reynolds JR (2018) Transparent wood smart windows: polymer electrochromic devices based on Poly(3,4-Ethylenedioxythiophene): poly(Styrene Sulfonate) electrodes. Chemsuschem 11(5):854-863
Lee H, Choi CH, Abbaspourrad A, Wesner C, Caggioni M, Zhu T, Weitz DA (2016) Encapsulation and enhanced retention of fragrance in polymer microcapsules. ACS Appl Mater Interf 8(6):4007-4013

Li J, Yu H, Sun Q, Liu Y, Cui Y, Lu Y (2010) Growth of $\mathrm{TiO}_{2}$ coating on wood surface using controlled hydrothermal method at low temperatures. Appl Surf Sci 256(16):5046-5050

Li W, Wang J, Wang X, Wu S, Zhang X (2007) Effects of ammonium chloride and heat treatment on residual formaldehyde contents of melamine-formaldehyde microcapsules. Coll Polym Sci 285(15):1691-1697

Li Y, Hui B, Lv M, Li J, Li G (2017) Inorganic-organic hybrid wood in response to visible light. J Mater Sci 53(5):3889-3898

Ma X, Wu YZ, Zhu HL (2013) The fire-retardant properties of the melamine-modified urea-formaldehyde resins mixed with ammonium polyphosphate. J Wood Sci 59(5):419-425

Ma Y, Zhu B (2009) Research on the preparation of reversibly thermochromic cement based materials at normal temperature. Cem Concr Res 39(2):90-94

Mao A, Hassan EB, Kim MG (2013) The effects of adding melamine at different resinsynthesis points of low mole ratio urea-melamineformaldehyde (UMF) resins. BioResources 8:5733-5748

Marvel CS, Elliott JR (1946) The structure of urea-formaldehyde resins. J Am Chem Soc 68(9):1681-1686

Németh B, Németh AS, Ujhidy A, Tóth J, Trif L, Gyenis J, Feczkó $\mathrm{T}$ (2018) Fully bio-originated latent heat storing calcium alginate microcapsules with high coconut oil loading. Sol Energy 170:314-322

No BY, Kim MG (2004) Syntheses and properties of low-level melamine-modified urea-melamine-formaldehyde resins. J Appl Polym Sci 93(6):2559-2569

No YB, Kim MG (2007) Evaluation of melamine-modified ureaformaldehyde resins as particleboard binders. J Appl Polym Sci 106(6):4148-4156

Pakdel H, Cyr PL, Riedl B, Deng J (2007) Quantification of urea formaldehyde resin in wood fibers using X-ray photoelectron spectroscopy and confocal laser scanning microscopy. Wood Sci Technol 42(2):133-148

Perez G, Allegro VR, Corroto M, Pons A, Guerrero A (2018) Smart reversible thermochromic mortar for improvement of energy efficiency in buildings. Constr Build Mater 186:884-891

Saputro EA, Al-Shannaq R, Farid MM (2019) Performance of metal and non-metal coated phase change materials microcapsules when used in compressed air energy storage system. Appl Ther Eng 157:113715

Shallcross RC, Zacharias P, Kohnen A, Korner PO, Maibach E, Meerholz K (2013) Photochromic transduction layers in organic memory elements. Adv Mater 25(3):469-476

Siimer K, Kaljuvee T, Pehk T, Lasn I (2009) Thermal behaviour of melamine-modified urea-formaldehyde resins. J Therm Anal Calorim 99(3):755-762

Toepke MW, Beebe DJ (2006) PDMS absorption of small molecules and consequences in microfluidic applications. Lab Chip 6(12):1484-1486

Wang L, Liu Y, Zhan X, Luo D, Sun X (2019) Photochromic transparent wood for photo-switchable smart window applications. J Mater Chem C 7(28):8649-8654

Wang W, Zhang W, Zhang S, Li J (2014a) Preparation and characterization of microencapsulated ammonium polyphosphate with UMF and its application in WPCs. Constr Build Mater 65:151-158

Wang X, Liu S, Chang H, Liu J (2014b) Sol-gel deposition of Tio2 nanocoatings on wood surfaces with enhanced hydrophobicity and photostability. Wood Fiber Sci 46(1):109-117

Wu K, Song L, Wang Z, Hu Y (2008) Microencapsulation of ammonium polyphosphate with PVA-melamine-formaldehyde resin 
and its flame retardance in polypropylene. Polym Adv Technol 19(12):1914-1921

Xu F, Zhao T, Yang T, Dong L, Guan X, Cui X (2016) Fabrication of folic acid functionalized $\mathrm{pH}$-responsive and thermosensitive magnetic chitosan microcapsules via a simple sonochemical method. Coll Surf A 490:22-29
Publisher's Note Springer Nature remains neutral with regard to jurisdictional claims in published maps and institutional affiliations. 